\title{
Coordinating Heterogeneous Distributed Energy Resources for Provision of Frequency Regulation Services*
}

\author{
Justin T. Hughes \\ University of Illinois \\ at Urbana-Champaign \\ hughes5@illinois.edu
}

\author{
Alejandro D. Domínguez-García \\ University of Illinois \\ at Urbana-Champaign \\ aledan@illinois.edu
}

\author{
Kameshwar Poolla \\ University of California, \\ Berkeley \\ poolla@berkeley.edu
}

\begin{abstract}
We discuss a framework for coordinating the response of distributed energy resources (DERs) connected to electric power distribution networks to provide frequency regulation services. These resources include plug-in electric vehicles, thermostatically controlled loads, and microturbines. In this framework, we consider an aggregator that participates in the real-time market by submitting an offer to provide frequency regulation services. If the offer is accepted, the aggregator needs to coordinate the response of a set of DERs. The DERs are compensated through bilateral contracts, the terms of which are negotiated in advance. The DER coordination problem the aggregator is faced with is cast as an optimal control problem, and we propose a bilayer framework to obtain a sub-optimal solution. In the first layer, we utilize model-predictive control techniques driven by regulation signal forecasts and parameter estimates to obtain a reference control signal for the DERs. A second control layer provides closed-loop regulation around the reference computed by the top layer, which minimizes the error that arises due to forecast error, plant-model mismatch, and the slower speed of the optimal control.
\end{abstract}

\section{Introduction}

Electric power systems are undergoing dramatic transformations in structure and functionality in response to the US DoE Smart Grid vision [1], and its European counterpart Electricity Networks of the Future [2]. These transformations are enabled by (i) the integra-

* Supported in part by EPRI and CERTS under sub-award 09206; PSERC S-52; NSF under Grants CNS-0931748, EECS-1129061, CPS-1239178, and CNS-1239274; the Republic of Singapore National Research Foundation through a grant to the Berkeley Education Alliance for Research in Singapore for the SinBerBEST Program. tion of new renewable generation resources (e.g., solar photovoltaic (PV) installations) and energy-storage capable loads (e.g., plug-in electric vehicles (PEVs)), and (ii) the increased reliance on advanced communications, which enables the active control of other types of energystorage capable loads such as thermostatically controlled loads (TCLs) (e.g., air conditioners, heat pumps, water heaters, and refrigerators).

These generation and controllable/storage-capable resources are commonly referred to as distributed energy resources (DERs). If properly coordinated, DERs provide new opportunities and added flexibility in the procurement of ancillary services such as frequency regulation and load following. For instance, PEVs and TCLs can be utilized to provide active power for up and down regulation services, e.g., energy peak-shaving during peak hours and load-leveling at night $[3,4,5]$. In order to enable this added functionality that these new technologies may provide, it is necessary to develop appropriate control mechanisms. In this paper, we discuss a framework for coordinating the response of DERs to provide frequency regulation and address a specific instance of this control design problem.

In this framework, we consider an aggregator that participates in a real-time market by submitting an offer to provide frequency regulation services. The aggregator does not own generation or storage assets. In the event an offer is accepted, it must coordinate the response of a set of heterogenous DERs, e.g., PEVs, TCLs, and microturbines, to provide the promised service. The DERs are compensated for participation through negotiated bilateral contracts. This compensation is agreed to $e x$ ante and may vary among the participating DERs. For example, for a microturbine, the aggregator is likely to take into account fuel cost when setting its monetary compensation; whereas for a collection of TCLs, the aggregator might consider inconvenience costs, e.g., the battery not being fully charged in a PEV when the owner 
needs to use it, or water being too cold in the case of a water heater.

The profit of the aggregator is the difference between the revenue obtained from selling the frequency regulation service in the real-time market and the costs incurred by the payments to the DERs and the penalties for not being able to follow the frequency regulation signal. Thus, since the revenue is determined by the market clearing price, which is fixed before service delivery, the aggregator maximizes its profit by minimizing the total payments to the DERs and the penalties incurred for not being able to follow the frequency regulation signal. We focus on this DER coordination problem faced by the aggregator and propose a bilayer control architecture to address it.

In the top control layer, the aggregator uses modelpredictive control (MPC) techniques to minimize the costs incurred when providing regulation during a fixed service interval at time-scales consistent with existing real-time regulation markets. ${ }^{1}$ The constraints in the optimal control problem include the inherent dynamics associated with the DERs power delivery process, their upper and lower power output limits, their upper and lower energy limits, as well as constraints on their ramping rates, i.e., the rate at which they can change their power output.

In the bottom layer, a closed-loop control, similar to that implemented in traditional automatic generation control (AGC) systems, regulates around the MPC solution calculated by the top layer. Separating fast and slow time scales when designing a controller is a wellestablished method for solving problems involving $\mathrm{dy}$ namics of differing speeds [8]. The authors of [9] have proposed replacing traditional AGC with MPC, but, to our knowledge, this paper is the first to propose the aforementioned bilayered approach to coordinating DERs. This approach benefits from both the speed of traditional AGC-like control and the foresight of MPC, giving results with impressive accuracy, while the amounts of computational power necessary to obtain such accurate solutions are limited.

There have been many recent papers that exploit the salient features of DERs in different ways, e.g., [10] uses receding horizon model predictive control of flexible loads for energy arbitrage. The framework proposed in [11] utilizes stochastic dynamic programming to arrive at a solution to a Markov process in which flexible loads respond to broadcasted prices. We will focus on papers that directly control DERs to provide frequency

\footnotetext{
${ }^{1}$ In a real-time market, the duration of the period over which the aggregator offers to provide the regulation service is typically five to ten minutes, and the offer needs to be submitted in advance, e.g., two periods before the actual service is to be provided if the offer is accepted $[6,7]$.
}

regulation services. For example, in [3], the authors use mean field games to control a very large population of homogeneous PEVs. In [12] and [13], the authors propose to use Markovian Decision Processes to, respectively, control heating, ventilation and air conditioning (HVAC) systems in commercial buildings, and deferrable loads such as pool pumps. Finally, in [5], the authors propose an allocation strategy for TCLs based on priority stacks. Many of these earlier works focus on a specific class of DERs, while we are interested in exploiting the distinct capabilities of diverse classes of DERs.

The formulation of the problem to be solved in the top layer of our architecture is similar to [14], in which the authors use MPC to coordinate frequency response of diverse types of resources. Here, we build on the framework introduced in that paper and make valuable contributions in several directions. First, we generalize the model of energy-limited DERs and introduce a cost function that explicitly takes into account the aggregator payments to the DERs. Second, we formalize the decision-making that the aggregator is faced with when coordinating the DER responses as a stochastic optimal control problem, and we show that in the perfect information case (i.e., the regulation signal is known a priori), the optimal control problem reduces to a linear program. Additionally, we go beyond the assumption of a perfect forecast and a two step prediction horizon in [14], and investigate longer time-horizon schemes that incorporate a forecasting technique the aggregator can use to handle imperfect information. Our architecture adds a second layer which improves tracking of fast moving regulation signals and decreases the computational complexity involved in finding a solution. Finally, we provide empirical evidence via synthetic simulations that use a mix of real and simulated data to show the effectiveness of these schemes and conduct parameter sensitivity studies. An aggregator can use similar studies to optimize the control and determine appropriate DER portfolios to profitably offer regulation services.

The authors of [15] and [16] propose an ambitious multi-level control framework for coordinating aggregations of commercial buildings. The nicely integrated result considers the interaction between the aggregator and the system operator/reserves market as well as the internal control of a single type of DER. In this framework, the reserve allocations for individual loads are calculated by the aggregator daily, while distributed controllers handle changes on the order of minutes and seconds. In contrast, we propose a framework in which the aggregator runs a fast, centralized control to coordinate the response. In our framework, the distributed controllers are not required to use predictive techniques for regulation. We believe this method is advantageous as the 
centralized controller is able to fully consider the entire pool of capability in real time, utilizing the strength of each heterogeneous DER as appropriate.

The remainder of this paper is organized as follows. In Section 2, we introduce models for each of the DER types considered, and formulate the DER coordination problem. In Section 3 we propose the top level of a control architecture that allows the aggregator to implement a solution to the DER coordination problem. In Section 4 we propose the second layer of the bilayer control architecture. The performance of this control architecture is showcased through simulation studies in Section 5. Concluding remarks and directions for future work are offered in Section 6.

\section{Problem Setting}

We first introduce the model that describes the dynamics associated with the power delivery process of the different types of DERs considered in this paper. We then capture the regulation cost associated with the DERs. Using these, we formulate the DER coordination problem faced by the aggregator.

\subsection{DER Power Delivery Model}

We assume the aggregator needs to coordinate various types of DERs, which could include small-rating conventional generators (commonly referred to as microturbines), commercial building HVAC systems, collections of PEVs or TCLs, and flexible industrial processes. We provide a single model - a generalization of the virtual battery model [17] — which can describe the behavior of any of these resources.

Let $P^{i}(t)=p^{i}(t)+p_{0}^{i}$ denote the power delivered by DER $i$ at time $t$, where $p_{0}^{i}$ is some nominal setting at which the DER is operating, and $p^{i}(t)$ is the amount of regulation power that this type of DER provides, and let $u^{i}(t)$ denote the rate of change of $p^{i}(t)$, i.e., $u^{i}(t)=\frac{\mathrm{d}}{\mathrm{d} t} p^{i}(t)$. Also, let $X^{i}(t)=x^{i}(t)+x_{0}^{i}$ denote the DER energy level at time $t$, where $x_{0}^{i}$ is some nominal energy level, and $x^{i}(t)$ is the variation in the DER energy level around $x_{0}^{i}$. Additionally, let $\bar{p}^{i}$ and $-p^{i}$ denote the maximum and minimum values of $p^{i}(t)$ as determined by the charge rate limits of the DER (e.g., maximum equipment power rating), and let $\bar{u}^{i}$ and $-\underline{u}^{i}$ denote the maximum and minimum values of $u^{i}(t)$ as determined by the DER ramping constraints (e.g., inertia). Finally, let $C^{i}$ denote the limit on up and down variation in $x^{i}(t)$ around $x_{0}^{i}$ as determined by capacity constraints (e.g., acceptable chemical charge or temperature range). Then, for the $i^{\text {th }}$ DER, we have

$$
\begin{gathered}
\frac{\mathrm{d}}{\mathrm{d} t} p^{i}(t)=u^{i}(t), \\
\frac{\mathrm{d}}{\mathrm{d} t} x^{i}(t)=-a^{i} x^{i}(t)-p^{i}(t), \\
-\underline{u}^{i} \leq u^{i}(t) \leq \bar{u}^{i}, \quad-\underline{p}^{i} \leq p^{i}(t) \leq \bar{p}^{i}, \quad\left|x^{i}(t)\right| \leq C^{i},
\end{gathered}
$$

where $a^{i} \geq 0$ captures the process of dissipation towards nominal energy, and $u^{i}(t)$ is controlled by the aggregator.

While we have considered symmetric constraints on $x^{i}(t)$, the formulation can be easily extended to the asymmetric case. Also, the dynamic model we adopt is consistent with those used in bulk power transmission systems to describe the regulation capabilities of units participating in AGC (see, e.g., [18]).

\subsection{DER Coordination Problem Formulation}

We assume that the aggregator does not own any DERs. To deliver the amount of frequency regulation stipulated through the clearing process of the real-time market, the aggregator needs to coordinate the response of a collection of $n$ heterogeneous DERs modeled as in Section 2.1.

We will assume that the DERs have agreed in advance to provide the service on behalf of the aggregator in exchange for some monetary compensation. For a given market-clearing price, in order to maximize its revenue, the aggregator needs to minimize its cost; thus, it needs to minimize the sum of the payments to the DERs and the penalty which it would incur if not able to follow the frequency regulation signal set by the regional transmission organization (RTO). In our formulation, the payments to the DERs are those associated with power and energy used for regulation provision. DERs may also receive a reservation payment based on capacity, independent of whether or not they are used for service provision. From the perspective of this formulation, these would be sunk costs which would not influence the coordination scheme.

Let $\pi_{1}^{i}$ denote the price per unit of power that the aggregator respectively pays DERs for providing power for both up and down regulation, and let $\pi_{2}^{i}$ denote the price per unit of deviation from baseline energy. Also, let $X$ denote the amount of power for up and down frequency regulation that the aggregator has offered in the real-time market. Additionally, let $\sigma X r(t), 0 \leq \sigma \leq 1$, where $r(t)$ is the value that the normalized regulation signal set by the RTO takes at time $t$, be the value of the signal that the aggregator needs to track at every time instant $t$; and let $\pi^{p}$ denote the price per unit of power that the aggregator incurs as a penalty if it does not track the signal. Finally, let $\left[t_{0}, t_{f}\right]$ be the time interval over 
which the aggregator provides regulation service. Then, given (1), the DER coordination problem faced by the aggregator is to find functions $u^{i}$ that minimize

$$
\begin{aligned}
& J\left(u^{1}, \cdots, u^{n}\right)= \\
& \quad \int_{t_{0}}^{t_{f}} L\left(p^{1}(t), x^{1}(t), \cdots, p^{n}(t), x^{n}(t), r(t)\right) d t
\end{aligned}
$$

where

$$
\begin{aligned}
& L\left(p^{1}(t), x^{1}(t), \cdots, p^{n}(t), x^{n}(t), r(t)\right)= \\
& \pi^{p}\left|\sigma X r(t)-\sum_{i=1}^{n} p^{i}(t)\right|+\sum_{k=1}^{n}\left(\pi_{1}^{i} p^{i}(t)+\pi_{2}^{i}\left|x^{i}(t)\right|\right) .
\end{aligned}
$$

In (2), while $t_{0}$ is likely to correspond to the beginning of the period over which the aggregator needs to provide frequency regulation, $t_{f}$ does not necessarily correspond to the time instant at which this period ends. In this regard, if the aggregator were to choose $t_{f}$ to exactly coincide with the time at which the frequency regulation period ends, then it would maximize its revenue for this period. However, if the aggregator were to participate in subsequent periods, this strategy might not be optimal; thus, the aggregator might decide to look ahead and consider a longer time horizon to better position itself.

In (3), a large penalty price, $\pi^{p}$, is assumed for error in tracking the regulation signal. A nonlinear imbalance penalty may be more accurate, but would greatly complicate the solution procedure. Regulation power is paid according to the amount of power used, which is negative for down regulation. Energy costs reflect the inconvenience cost of deviating from the baseline value, e.g., uncomfortable temperature, insufficient battery charge, or no hot water. This function could be generalized to include mileage payments, which account for increased maintenance costs due to cycling the equipment.

The normalized regulation signal, $r(t)$, is computed in real-time by the RTO based on the frequency error and inter-area power exchange errors (see, e.g., [18]); thus, this signal is not known a priori. This uncertainty adds a crucial complicating factor for the aggregator. In the following two sections, we provide a bilayer architecture that the aggregator can use to provide a solution to the DER coordination problem. In the top layer, all costs are considered and a forecast of the regulation signal will be used; the bottom layer regulates around the top layer solution in order to minimize short-term tracking error.

\section{Top Layer DER Coordination Scheme}

We first discretize the DER coordination problem as defined in (1) - (3), and provide an exact solution for the case where the regulation signal is known a priori to the aggregator. We then discuss the effects of uncertainty and long time horizons.

\subsection{Perfect Information, Fixed-Horizon}

We will show that the DER coordination problem reduces to a linear program under perfect information with a fixed service interval. To this end, define $x(t)=\left[p^{1}(t), x^{1}(t), \cdots, p^{n}(t), x^{n}(t)\right]^{T}$, and $u(t)=$ $\left[u^{1}(t), \cdots, u^{n}(t)\right]^{T}$; then, the differential equations in (1) can be written as

$$
\dot{x}(t)=\widetilde{A} x(t)+\widetilde{B} u(t)
$$

where

$$
\begin{aligned}
& \widetilde{A}^{i}=\left[\begin{array}{cc}
0 & 0 \\
-1 & -a^{i}
\end{array}\right] \quad \widetilde{B}^{i}=\left[\begin{array}{ccc} 
& \hat{e}^{i} & \\
0 & \cdots & 0
\end{array}\right] \\
& \widetilde{A}=\left[\begin{array}{cccc}
\widetilde{A}^{1} & 0 & \cdots & 0 \\
0 & \widetilde{A}^{2} & \cdots & 0 \\
\vdots & \vdots & \ddots & \vdots \\
0 & 0 & \cdots & \widetilde{A}^{n}
\end{array}\right] \quad \widetilde{B}=\left[\begin{array}{c}
\widetilde{B}^{1} \\
\widetilde{B}^{2} \\
\vdots \\
\widetilde{B}^{n}
\end{array}\right]
\end{aligned}
$$

with $\hat{e}^{i}$ being the unit row vector pointing in dimension $i$. This model can be replaced by a discrete-time statespace model of the form

$$
x_{k}=A x_{k-1}+B u_{k-1}, k=1, \ldots, N,
$$

where $N=\left(t_{f}-t_{0}\right) / \Delta T_{1} \in \mathbb{N}, x_{k}=x\left(k \Delta T_{1}+t_{0}\right), u_{k}=$ $u\left(k \Delta T_{1}+t_{0}\right), A=I+\widetilde{A} \Delta T_{1}$, and $B=\widetilde{B} \Delta T_{1}$. As is standard, the discretization error can be made negligible by choice of sampling time $\Delta T_{1}$.

The constraints in (1) can also be compactly written in matrix form as follows:

$$
E_{x} x_{k} \leq F_{x}, \quad E_{u} u_{k} \leq F_{u},
$$

where

$$
\begin{aligned}
E_{x} & =\left[\hat{e}^{1},-\hat{e}^{1}, \hat{e}^{2},-\hat{e}^{2}, \cdots, \hat{e}^{2 n-1},-\hat{e}^{2 n-1}, \hat{e}^{2 n},-\hat{e}^{2 n}\right]^{T} \\
F_{x} & =\left[\bar{p}^{1}, \underline{p}^{1}, C^{1}, C^{1}, \cdots, \bar{p}^{n}, \underline{p}^{n}, C^{n}, C^{n}\right]^{T} \\
E_{u} & =\left[\hat{e}^{1},-\hat{e}^{1}, \cdots, \hat{e}^{n},-\hat{e}^{n}\right]^{T} \\
F_{u} & =\left[\bar{u}^{1}, \underline{u}^{1}, \cdots, \bar{u}^{n}, \underline{u}^{n}\right]^{T} .
\end{aligned}
$$

The cost functional in (2) can also be discretized as:

$$
J(u)=\Delta T_{1} \sum_{k=1}^{N}\left(Q_{1} x_{k}+\left\|Q_{2} x_{k}+R r_{k}\right\|_{1}\right),
$$

where $r_{k}=r\left(k \Delta T_{1}+t_{0}\right), k=1, \ldots, N$, and

$$
\begin{aligned}
Q_{1} & =\left[0 \hat{e}^{1}, \pi_{1}^{1} \hat{e}^{1}, 0 \hat{e}^{2}, \cdots, \pi_{1}^{n} \hat{e}^{2 n-1}, 0 \hat{e}^{2 n}\right]^{T} \\
Q_{2} & =\left[-\pi^{p} \sum_{i=1}^{n} \hat{e}^{2 i-1}, 0 \hat{e}^{1}, \pi_{2}^{1} \hat{e}^{2}, \cdots, 0 \hat{e}^{2 n-1}, \pi_{2}^{n} \hat{e}^{2 n}\right]^{T} \\
R & =\left[\pi^{p} \sigma X, 0, \cdots, 0\right]^{T}
\end{aligned}
$$


Combining (4) - (8), we can formulate the optimization problem

$$
\begin{array}{cl}
u_{k}^{*}=\underset{u}{\operatorname{argmin}} & \Delta T_{1} \sum_{k=1}^{N}\left(Q_{1} x_{k}+\left\|Q_{2} x_{k}+R r_{k}\right\|_{1}\right) \\
\text { subject to } & E_{x} x_{k} \leq F_{x} \\
& E_{u} u_{k} \leq F_{u}, \\
& x_{k}=A x_{k-1}+B u_{k-1},
\end{array}
$$

the solution of which can be used to solve the DER coordination problem as defined by (1) - (3).

Using the technique laid out in the Appendix, the optimization problem in (9) can be cast as a linear program of the form

$$
\begin{array}{ll}
\underset{y}{\operatorname{minimize}} & f^{T} y \\
\text { subject to } & G y \leq h,
\end{array}
$$

where $f \in \mathbb{R}^{(3 n+1) N}, \quad y \in \mathbb{R}^{(3 n+1) N}, \quad G \in$ $\mathbb{R}^{(3 n+1) N \times(10 n+2) N}, \quad h \in \mathbb{R}^{(10 n+2) N}$. This linear program can be solved using any of a number of well documented linear programming algorithms in the literature (see, e.g., [19, 20]).

\subsection{Imperfect Information, Receding Horizon}

To solve the DER coordination problem via (10), it is necessary to have complete information of the values that the regulation signal $r(t)$ takes for all $t=$ $t_{0}+k \Delta T_{1}, k=1, \ldots, N$. Next, we propose an MPCbased solution to the DER coordination problem when $r(t)$ is not known in advance.

We use the subscript $k+l \mid l$ to denote an estimate, made at time $t_{l}=t_{0}+l \Delta T_{1}$, of the value that a variable takes at time $t_{k+l}=t_{0}+(k+l) \Delta T_{1}$, e.g., $n_{k+l \mid l}$ denotes the estimate of the regulation signal $k$ steps ahead of time $t_{0}+l \Delta T_{1}$ (this, and other notation used here was adopted from [21]). With this notation we can write an optimization program similar to (9), but with an arbitrary starting point and no requirement of perfect knowledge of the future:

$$
\begin{gathered}
u_{k}^{*}=\underset{u}{\operatorname{argmin}} \Delta T_{1} \sum_{k=1}^{N}\left(Q_{1} x_{l+k \mid l}+\left\|Q_{2} x_{l+k \mid l}+R r_{l+k \mid l}\right\|_{1}\right) \\
\text { subject to } E_{x} x_{l+k \mid l} \leq F_{x} \\
E_{u} u_{l+k \mid l} \leq F_{u} \\
x_{l+k \mid l}=A x_{l+k-1 \mid l}+B u_{l+k-1 \mid l}
\end{gathered}
$$

with $E_{x}, F_{x}, E_{u}$, and $F_{u}$ as in (6); and $Q_{1}, Q_{2}$, and $R$ as in (8). This optimization can also be rewritten in the form in (10).

Suppose the solution to (11) was calculated with $l=0$. This is equivalent to (9) with an arbitrary forecast.
This solution only depends on the current state $x_{0 \mid 0}$ and an estimate of future values to calculate the optimal control for the present time and next $N-1$ time steps. The first calculated optimal control input, denoted $u_{0 \mid 0}^{*}$, is then applied. At time $t_{0}+\Delta T_{1}$, the system state may not have evolved as predicted due to an inaccurate forecast of $r\left(t_{0}+\Delta T_{1}\right)$, incorrect system parameters, or unmodeled disturbances, i.e., $x_{1 \mid 0} \neq x_{1 \mid 1}, r_{1 \mid 0} \neq r_{1 \mid 1}$. Thus, in order to obtain a better solution, this new information should be taken into account.

At the next time step we assign $l \leftarrow l+1$ and update $x_{l \mid l}$ with new measurements, and the forecast of $r(t)$ with the latest information. We also assign $N \leftarrow N-1$ to avoid making choices that would create higher costs for $t \leq t_{f}$ in exchange for even lower costs when $t>t_{f}$. The problem is solved again, giving us a new control plan taking the latest information into account. The first optimal control input from this optimization is applied at this time step, and so on. The procedure continues until $l=N-1$. Then, at the following time step, we have that $t=t_{f}$, thus arriving to the final solution.

If the aggregator continues providing frequency regulation beyond time $t_{f}$ because it has cleared subsequent markets, it is not desirable for the aggregator to be left in a position where it cannot, or cannot profitably, meet future obligations. For this reason the aggregator would likely prefer to use a technique that takes a more farsighted view. This can be done by performing the steps described in the previous paragraph without decrementing $N$. The procedure continues until some arbitrary time step $l=M, M>N$. This technique is known as receding horizon control because as time progresses, the optimization window is also extended. A receding horizon will be used in the numerical examples presented in the next section.

As future values of $r(t)$ are unknown, forecasts must be used. On a second by second basis the regulation signal does not change much, so in the short term we expect future values of the regulation signal to be similar to the present value. However, the current value of the regulation signal tells us practically nothing about its value in the distant future; in this case it is best to predict the mean value. In the medium term it would be logical to interpolate between the present value and the mean. With appropriate choice of $\alpha_{1}$, linear interpolation,

$$
r_{k+l \mid l}=r_{l \mid l} \cdot \max \left(1-\alpha_{1} k \Delta T_{1}, 0\right), k \geq 1, \alpha_{1}>0,
$$

was found to have acceptable results.

Because the uncertainty of the forecasts increases with the prediction horizon, the objective function used by the predictive controller can be multiplied by a factor which de-weights values that are further into the future, 
i.e.,

$$
\begin{aligned}
& J(u)=\Delta T_{1} \sum_{k=1}^{N}\left(e ^ { - \alpha _ { 2 } k \Delta T _ { 1 } } \left(Q_{1} x_{l+k \mid l}\right.\right. \\
&\left.\left.\quad\left\|Q_{2} x_{l+k \mid l}+R r_{l+k \mid l}\right\|_{1}\right)\right) .
\end{aligned}
$$

\section{Bottom Layer Regulation Provision}

We next propose a second control layer, which provides closed-loop control so as to minimize the error that arises due to forecast error, plant-model mismatch, and the slower speed of the optimal control. Constraints are given special consideration, which removes the possibility of violations.

\subsection{Controller Formulation}

Conventional AGC, which is typically implemented using proportional-integral (PI) control, is used to coordinate participants in frequency regulation markets [22]. It is natural to apply a similar closed-loop control scheme to the problem at hand. Unlike predictive control, PI control is simple and can be performed very quickly. The controller has a single state variable, $z_{k}$, which is proportional to the integral of the tracking error. Let $p_{k+1}^{i \text { MPC }}$ denote the optimal power value for unit $i$ at time step $k+1$ as calculated by the most recent solution from the MPC-based top layer control, and let $p_{k+1}^{i *}$ denote the optimal power value for unit $i$ at time step $k+1$ as calculated by the bottom layer control before considering the constraints in 1 . Finally, let $u_{k}^{i}$ denote the control value to be sent to unit $i$ at time step $k$. Then, the controller can be formulated as follows:

$$
\begin{aligned}
z_{k+1} & =z_{k}+\Delta T_{2} \eta_{2}\left(\sigma X r_{k}-\sum_{i=1}^{n} p_{k}^{i}\right) \\
p_{k+1}^{i *} & =\beta^{i}\left(\eta_{1}\left(\sigma X r_{k}-\sum_{i=1}^{n} p_{k}^{i}\right)+z_{k+1}\right)+p_{k+1}^{i \mathrm{MPC}} \\
u_{k}^{i} & =\left[\frac{p_{k+1}^{i *}-p_{k}^{i}}{\Delta T_{2}}\right]^{+}
\end{aligned}
$$

where $\Delta T_{2}<\Delta T_{1}$ is the time step of the bottom layer control, $\eta_{2}$ is the integral gain, $\eta_{1}$ is the proportional gain, $\beta^{i}$ is the participation factor of DER $i$ with $\sum_{i=1}^{N} \beta^{i}=1$, and $[\cdot]^{+}$indicates projection onto the interval

$$
\begin{aligned}
\zeta^{i}= & {\left[\max \left(-\underline{u}^{i}, \frac{-\underline{p}^{i}-p_{k}^{i}}{\Delta T_{2}}, \frac{\breve{p}_{\dot{k}^{*}}^{i}-p_{k}^{i}}{\Delta T_{2}}\right),\right.} \\
& \left.\min \left(\bar{u}^{i}, \frac{\bar{p}^{i}-p_{k}^{i}}{\Delta T_{2}}, \frac{\hat{p}_{\hat{k}^{*}}^{i}-p_{k}^{i}}{\Delta T_{2}}\right)\right],
\end{aligned}
$$

which ensures all constraints in 1 are satisfied. The variables $\check{p}_{\check{k}^{*}}$ and $\hat{p}_{\hat{k}^{*}}$ are lower and upper bounds on power derived from capacity constraints; the exact definition and the procedure for calculating them will be given in the Section 4.2. We reset the integral control, i.e., $z_{k}=0$, when a new top layer solution is found. This control can also be used without the top layer by fixing $p_{k+1}^{i \mathrm{MPC}}=0$ and not resetting $z_{k}$.

Participation factor selection must balance optimality and complexity. The simplest possible values of $\beta^{i}$ would be the constants $1 / n$. The optimal values would be time-varying and would consider the costs, current values of state variables, and limits of each unit. The computation effort required to arrive at such a solution would likely be more effective if it were spent working on solving the MPC problem more quickly. A compromise solution would use a heuristic to update $\beta^{i}$ infrequently, likely based on the slow optimization solution.

The ramp and rate limit terms straightforwardly limit the control input at the current step and the power at the following time step to be within the defined limits. The control input takes at least two time steps to affect the state of charge, and control action could potentially need to be taken even further in advance in order to avoid constraint violations. Thus, determining these limits is more difficult; their values are derived in the following subsection.

\subsection{Capacity Constraints}

For a single DER, the dynamic equations in (1) can be replaced by a discrete-time state-space model of the form

$$
X_{k}=A X_{k-1}+B u_{k-1},
$$

where $X_{k}=\left[p\left(k \Delta T_{2}+t_{0}\right), x\left(k \Delta T_{2}+t_{0}\right)\right]^{T}, u_{k}=u\left(k \Delta T_{2}+\right.$ $\left.t_{0}\right), A=I+\widetilde{A} \Delta T_{2}$, and $B=\widetilde{B} \Delta T_{2}$.

We consider the bound $x \leq C$. If $x=C$, we must enforce the constraint $p \geq-a C$. Because $\bar{u}$ limits how quickly we can increase $p$, if $p<-a C$ we must also make sure it is possible to ramp to $p=-a C$ before $x>C$. We can find the limits on $u$ by starting at $\check{X}_{0}=[-a C C]^{T}$, and working the dynamics (14) backwards with $u_{k}=\bar{u}$ using the equation

$$
\check{X}_{k+1}=A^{-1}\left(\check{X}_{k}-B \bar{u}\right),
$$

with $k$ increasing until $\check{p}_{k} \leq-\underline{p}$ or $\check{x}_{k} \leq-C$, where $\check{X}_{k}=$ $\left[\begin{array}{ll}\check{p}_{k} & \check{x}_{k}\end{array}\right]^{T}$. This procedure only needs to be done once; the values can be stored and used at each time step in the future.

Then, at each time step, calculate

$$
\begin{aligned}
& \check{k}^{*}=\underset{k}{\arg \min } \quad \check{x}_{k}-\left(x-\Delta T_{2}(p+a x)\right) \\
& \text { subject to } \check{x}_{k} \geq\left(x-\Delta T_{2}(p+a x)\right) \text {. }
\end{aligned}
$$


This solution will always exist and be unique. Although this may look like a computationally difficult optimization problem, it is not; since $\breve{x}_{k}$ is monotonous, its value can be obtained via a lookup table. The limit on $u$ due to the constraint $x \leq C$ is then

$$
\frac{\check{p}_{\check{k}^{*}}-p_{k}}{\Delta T_{2}} .
$$

$\hat{p}_{\hat{k}^{*}}$ is calculated similarly, starting at $\hat{X}_{0}=\left[\begin{array}{ll}a C & -C\end{array}\right]^{T}$, and working the dynamics (14) backwards with $u_{k}=-\underline{u}$.

\section{Case Studies}

We test the performance of the DER coordination architecture introduced in the previous sections. We first describe the features of the dataset used in all case studies. Then, we present base-case simulation results.

\subsection{Dataset}

We utilize "Normalized Dynamic and Traditional Regulation Signals" from PJM for the period January 1-18, 2013. This data is available at [23], and includes two regulation signals_- RegD, a fast response signal, and $\operatorname{Reg} A$, a filtered version of RegD for slower ramping generators; both signals are updated every $2 \mathrm{~s}$.

Figure 1 shows a representative segment of the aforementioned data. We choose the fast signal RegD for our studies because DERs are expected to have faster ramping rates than conventional generators, and because storage devices work best with a zero-mean signal. Average cost and capability data for the same period is calculated using data available at [24]. From January 1-18, 2013, the average cost of regulation capacity was $\$ 14.3 / \mathrm{MWh}$, and $947 \mathrm{MW}$ of capacity was dispatched.

\subsection{Base Case}

A case study involving two units was investigated. The first unit has low ramp rate limits, but a low cost and high energy capacity. The second unit is able to ramp its power consumption more quickly, but is more expensive and has a lower energy capacity. The parameters for the studies are given in Table 1 . The penalty price $\pi^{p}$ was chosen to be ten times the PJM average capability clearing price. It was assumed the aggregator's dispatched capability made up about $2 \%$ of the total market. Time steps $\Delta T_{1}$ and $\Delta T_{2}$ are $20 \mathrm{~s}$ and $2 \mathrm{~s}$, respectively, and the prediction horizon $T$ is set to $5 \mathrm{~min}$. We use receding horizon control to calculate $u^{*}$ for $60 \mathrm{~min}$. We select a representative segment of $\mathrm{RegD}$ for the regulation signal $r_{k}$. The other parameters were selected to show different types of behavior that can arise.

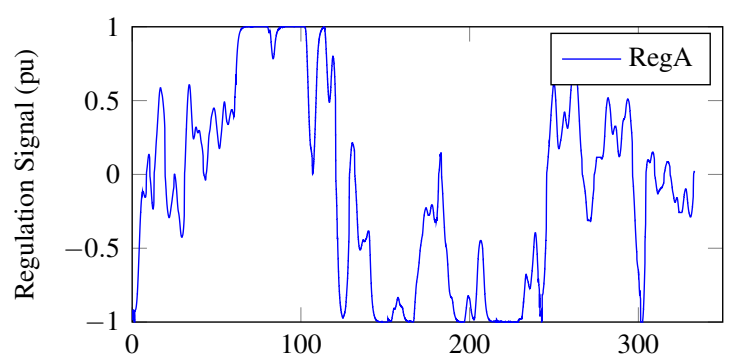

Time (min)

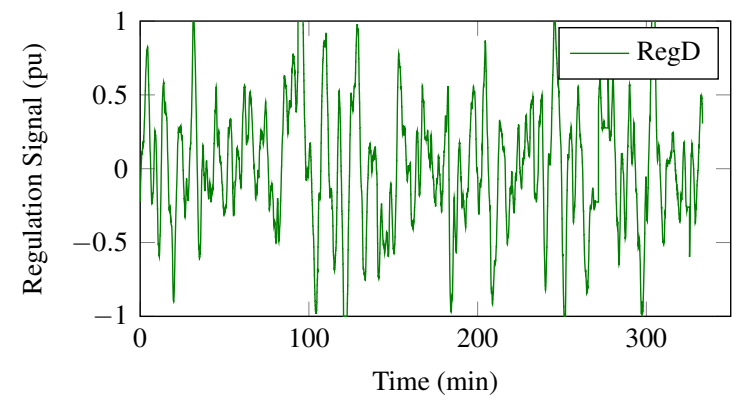

Figure 1. Segment of PJM regulation data. RegD is the fast dynamic response signal. RegA is a filtered version for slower responding units.

Fig. 2 illustrates the behavior of the fast bottom layer controller, the slow top layer, and the bilayer control. We observe that the fast control better tracks the small variations in $r(t)$, whereas the slow control uses prediction to spend less time bounded by energy constraints. The bilayer controller combines these two advantages. As expected, results in Table 2 show that the top layer performed better than the bottom at equal time scales. If the MPC is constrained to run more slowly than the PI control, its performance decreases, but it still outperforms the bottom layer running ten times faster. However, combining the two methods results in cost values lower than the slow top layer, while requiring significantly less computation power than the fast top layer control.

Both the fast and slow control loops take measures to ensure solutions are feasible. However, feasibility problems are encountered when the time scales are mixed. The MPC problem can be given an initial condition which is feasible on the fast time scale, but unfeasible on the slow time scale. This must be resolved by requiring all fast control solutions to be slow time feasible, or by relaxing constraints on the slow time solution. 

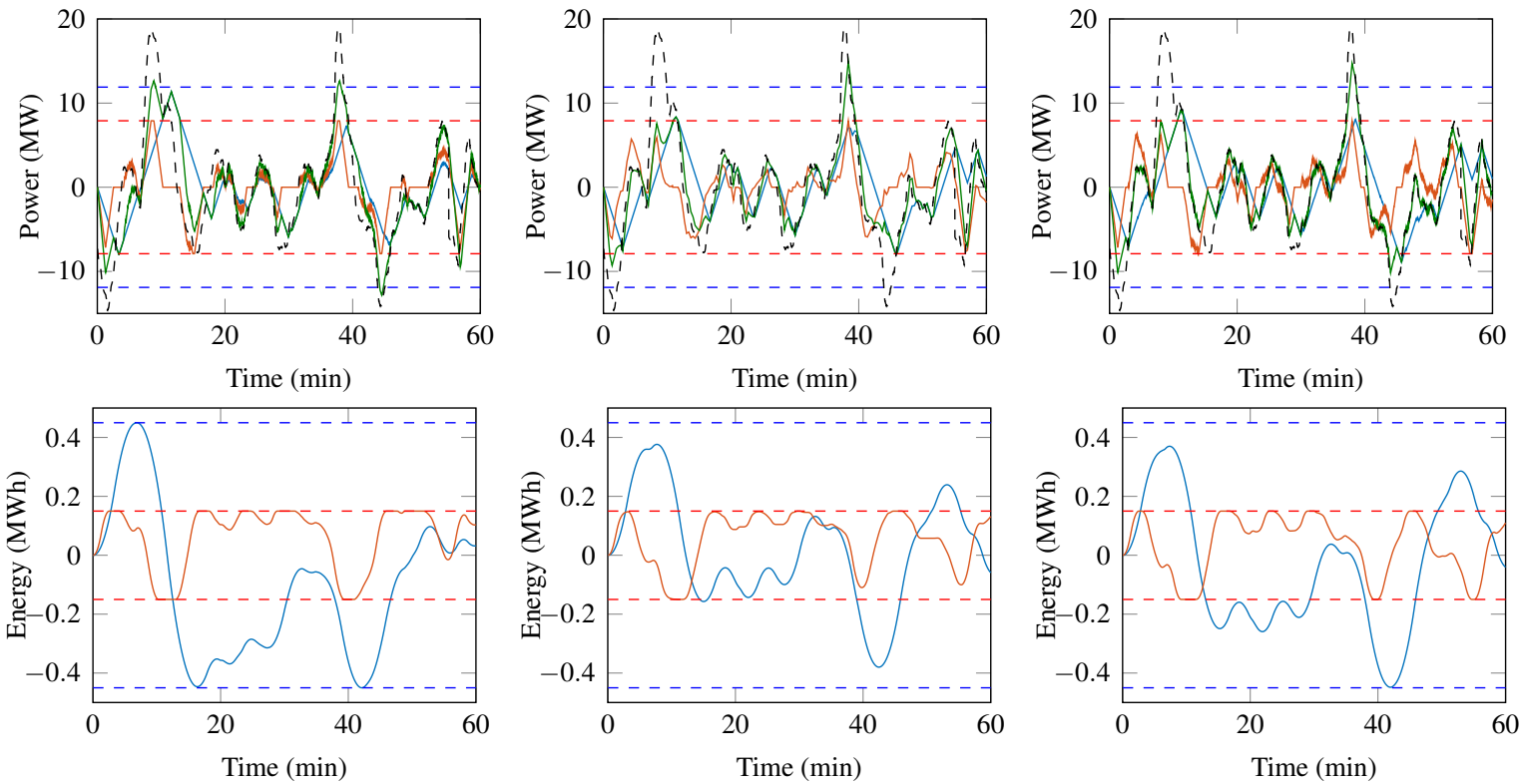

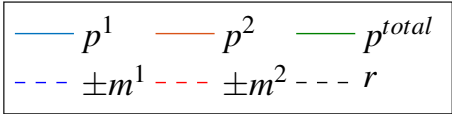

$$
\begin{aligned}
& -x^{1}-x^{2} \\
& --- \pm C^{1---} \pm C^{2}
\end{aligned}
$$

Figure 2. Numerical simulation results. Left to right: Bottom layer control, Top layer control, Bilayer control. Top to bottom: power, state of charge.

Table 1. Case study parameters

\begin{tabular}{clcc}
\hline Parameter & Description & Value & Unit \\
\hline$\pi_{1}^{1}$ & Regulation Price & 14.3 & $\$ / \mathrm{MW}$ \\
$\pi_{1}^{2}$ & Regulation Price & 42.9 & $\$ / \mathrm{MW}$ \\
$\pi_{2}^{1}, \pi_{2}^{2}$ & Energy Price & 0 & $\$ / \mathrm{MWh}$ \\
$\pi^{p}$ & Imbalance Price & 143 & $\$ / \mathrm{MWh}$ \\
$\bar{u}^{1}, \underline{u}^{1}$ & Ramp Limit & 0.04 & $\mathrm{MW} / \mathrm{s}$ \\
$\bar{u}^{2}, \underline{u}^{2}$ & Ramp Limit & 0.096 & $\mathrm{MW} / \mathrm{s}$ \\
$\bar{p}^{1}, \underline{p}^{1}$ & Regulation Limit & 11.9 & $\mathrm{MW}$ \\
$\bar{p}^{2}, \bar{p}^{2}$ & Regulation Limit & 7.9 & $\mathrm{MW}$ \\
$C^{1}$ & Storage Energy Limit & 0.45 & $\mathrm{MWh}$ \\
$C^{2}$ & Storage Energy Limit & 0.15 & $\mathrm{MWh}$ \\
$a^{1}, a^{2}$ & Dissipation Constant & 0 & $\mathrm{~s}-1$ \\
$\sigma X$ & Regulation Signal Magnitude & 18.9 & $\mathrm{MW}$ \\
$T$ & Prediction Horizon & 5 & $\mathrm{~min}$ \\
\hline
\end{tabular}

\subsection{Sensitivity}

Here, we explore the sensitivity of the control scheme total cost with regard to time step size, controller gains, and forecasting parameters.

Base case controller parameters were optimized using a 12 hour long segment of the PJM regulation signal as training data. Fig. 3 shows that the optimal participation factors require DER 1, which has a lower regulation price, to participate less than DER 2. However, DER 2 still has significant participation despite its higher price due to its faster ramping ability. Fig. 4 and Fig. 5 il-
Table 2. Base case total cost for different control strategies

\begin{tabular}{ccc}
\hline Control & Time-Step & Total Cost $(\$)$ \\
\hline Bottom Layer & $20 \mathrm{~s}$ & 584.39 \\
Bottom Layer & $2 \mathrm{~s}$ & 480.58 \\
Top Layer & $20 \mathrm{~s}$ & 470.37 \\
Top Layer & $2 \mathrm{~s}$ & 362.10 \\
Bilayer & $2 \mathrm{~s} / 20 \mathrm{~s}$ & 387.13 \\
\hline
\end{tabular}

lustrate the sensitivity of cost to controller gains and forecast parameters, respectively. Even with a $12 \mathrm{~h} \mathrm{sim}$ ulation period, these functions are non-convex, making optimization difficult. Multi-start methods were used to attempt to locate the global minimum.

\section{Conclusions}

We have discussed a framework for an aggregator to coordinate the amount of power provided by a collection of heterogeneous DERs for providing up and down frequency regulation services. By coordinating the response of the DERs, the aggregator can sell this service in real-time regulation markets.

We have shown that the DER coordination problem that the aggregator is faced with can be cast as a 


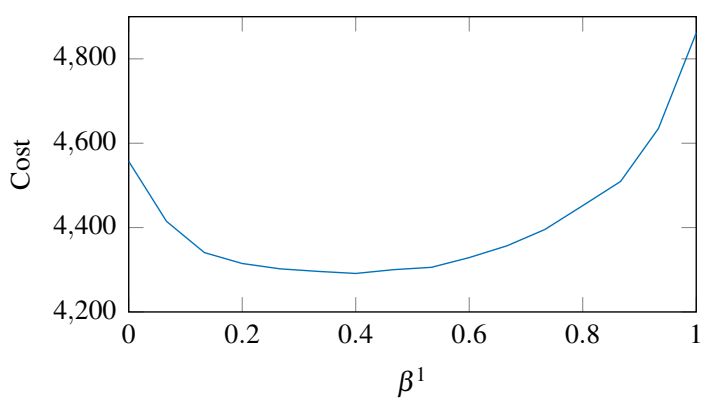

Figure 3. Sensitivity of operating costs to participation factors.

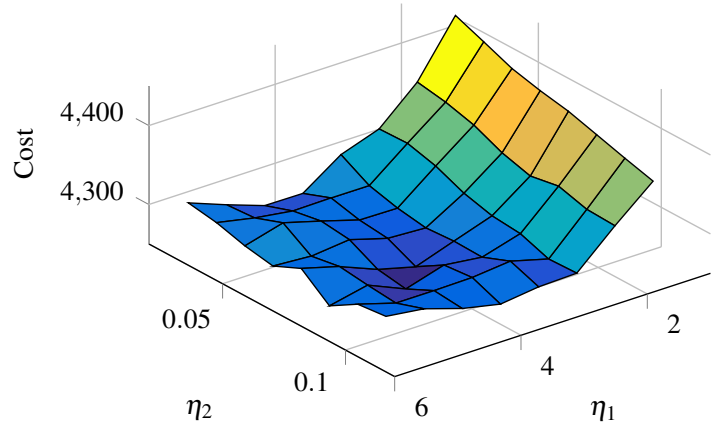

Figure 4. Sensitivity of operating costs to bottom layer controller parameters.

stochastic optimal control problem. We have provided a bilayer control scheme that enables the aggregator to solve this problem by using a slow but accurate predictive control techniques while also responding quickly to second-to-second variations in the regulation signal.

A related problem that is worthy of future exploration is the decision-making process that the aggregator uses under this framework to choose the capability and price to offer in the market under the uncertainty of DER parameters and regulation signal frequency content.

\section{Appendix}

The following definitions are used in the process of casting (9) as a standard linear program:

$$
\begin{aligned}
\mathcal{X} & =\left[\begin{array}{llll}
x_{1}^{T} & x_{2}^{T} & \ldots & x_{N}^{T}
\end{array}\right]^{T} \quad \mathcal{R}=\left[\begin{array}{c}
R r_{1} \\
\vdots \\
R r_{N}
\end{array}\right] \Delta T_{1} \\
\mathcal{U} & =\left[\begin{array}{llll}
u_{0}^{T} & u_{1}^{T} & \ldots & u_{N-1}^{T}
\end{array}\right]^{T} \quad \\
\mathcal{A} & =\left[\begin{array}{c}
A \\
A^{2} \\
\vdots \\
A^{N}
\end{array}\right] \quad \mathcal{B}=\left[\begin{array}{cccc}
B & 0 & \ldots & 0 \\
A B & B & \ldots & 0 \\
\vdots & \ddots & \ddots & \vdots \\
A^{N-1} B & \ldots & A B & B
\end{array}\right]
\end{aligned}
$$

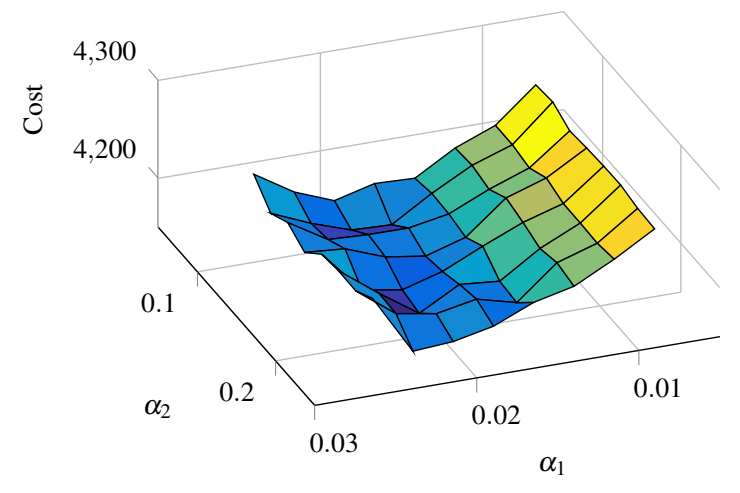

Figure 5. Sensitivity of operating costs to forecast parameters.

$$
\begin{gathered}
\mathcal{E}_{x}=\left[\begin{array}{cccc}
E_{x} & 0 & \ldots & 0 \\
0 & E_{x} & \ddots & \vdots \\
\vdots & \ddots & \ddots & 0 \\
0 & \ldots & 0 & E_{x}
\end{array}\right] \quad \mathcal{E}_{u}=\left[\begin{array}{cccc}
E_{u} & 0 & \ldots & 0 \\
0 & E_{u} & \ddots & \vdots \\
\vdots & \ddots & \ddots & 0 \\
0 & \ldots & 0 & E_{u}
\end{array}\right] \\
\mathcal{Q}_{1}=\left[\begin{array}{cccc}
Q_{1} & 0 & \ldots & 0 \\
0 & Q_{1} & \ddots & \vdots \\
\vdots & \ddots & \ddots & 0 \\
0 & \ldots & 0 & Q_{1}
\end{array}\right] \Delta T_{1} \mathcal{F}_{x}=\left[\begin{array}{c}
F_{x} \\
\vdots \\
F_{x}
\end{array}\right] \\
\mathcal{Q}_{2}=\left[\begin{array}{cccc}
Q_{2} & 0 & \ldots & 0 \\
0 & Q_{2} & \ddots & \vdots \\
\vdots & \ddots & \ddots & 0 \\
0 & \ldots & 0 & Q_{2}
\end{array}\right] \Delta T_{1} \mathcal{F}_{u}=\left[\begin{array}{c}
F_{u} \\
\vdots \\
F_{u}
\end{array}\right] .
\end{gathered}
$$

With these definitions, the problem can be written without the summation:

$$
\begin{array}{cl}
\underset{\mathcal{U}}{\operatorname{minimize}} & \mathbf{1}^{T} \mathcal{Q}_{1} \mathcal{X}+\left\|\mathcal{Q}_{2} \mathcal{X}+\mathcal{R}\right\|_{1} \\
\text { subject to } & \mathcal{E}_{X} \mathcal{X} \leq \mathcal{F}_{X} \\
& \mathcal{E}_{U} \mathcal{U} \leq \mathcal{F}_{U} \\
& \mathcal{X}=\mathcal{A} x_{0}+\mathcal{B U}
\end{array}
$$

Introduce the variable $Z_{x}$ to bound $\mathcal{Q}_{2} \mathcal{X}+\mathcal{R}$, which removes the norm in the objective function, and the problem becomes

$$
\begin{array}{cl}
\underset{\mathcal{U}, Z_{x}}{\operatorname{minimize}} & \mathbf{1}^{T} \mathcal{Q}_{1} \mathcal{X}+\mathbf{1}^{T} Z_{x} \\
\text { subject to } & \mathcal{E}_{X} \mathcal{X} \leq \mathcal{F}_{X} \\
& \mathcal{E}_{U} \mathcal{U} \leq \mathcal{F}_{U} \\
& \mathcal{X}=\mathcal{A} x_{0}+\mathcal{B U} \\
& -Z_{x} \leq \mathcal{Q}_{2} \mathcal{X}+\mathcal{R} \leq Z_{x} .
\end{array}
$$


Eliminating $\mathcal{X}$ by plugging in the dynamic equations gives us

$$
\begin{array}{cl}
\underset{\mathcal{U}, Z_{x}}{\operatorname{minimize}} & \mathbf{1}^{T} \mathcal{Q}_{1} \mathcal{A} x_{0}+\mathbf{1}^{T} \mathcal{Q}_{1} \mathcal{B U}+\mathbf{1}^{T} Z_{x} \\
\text { subject to } & \mathcal{E}_{x} B \mathcal{U} \leq \mathcal{F}_{x}-\mathcal{E}_{x} \mathcal{A} x_{0} \\
& \mathcal{E}_{u} \mathcal{U} \leq \mathcal{F}_{u} \\
& -Z_{x} \leq \mathcal{Q}_{2}\left(\mathcal{A} x_{0}+\mathcal{B U}\right)+\mathcal{R} \leq Z_{x} .
\end{array}
$$

To create a single unknown variable and a single and inequality, we define

$$
\begin{array}{rlr}
f & =\left[\begin{array}{ll}
\mathbf{1}^{T} \mathcal{Q}_{1} \mathcal{B} & \mathbf{1}^{T}
\end{array}\right]^{T}, & y=\left[\begin{array}{ll}
\mathcal{U} & Z_{x}
\end{array}\right]^{T}, \\
G=\left[\begin{array}{cc}
-\mathcal{Q}_{2} \mathcal{B} & -I \\
\mathcal{Q}_{2} \mathcal{B} & -I \\
\mathcal{E}_{u} & 0 \\
\mathcal{E}_{x} \mathcal{B} & 0
\end{array}\right], & h=\left[\begin{array}{c}
\mathcal{Q}_{2} \mathcal{A} x_{0}+\mathcal{R} \\
-\mathcal{Q}_{2} \mathcal{A} x_{0}-\mathcal{R} \\
\mathcal{F}_{u} \\
\mathcal{F}_{x}-\mathcal{E}_{x} \mathcal{A} x_{0}
\end{array}\right] .
\end{array}
$$

Note the term $\mathbf{1}^{T} \mathcal{Q}_{1} \mathcal{A} x_{0}$ is ignored as it is constant with respect to the decision variables. The problem is then in the form of a standard linear program (10).

\section{References}

[1] “U.S.DoE — Smart Grid," http://www.oe.energy.gov/ smartgrid.htm.

[2] "European technology platform for the electricity networks of the future," http://www.smartgrids.eu.

[3] D. S. Callaway and I. A. Hiskens, "Achieving controllability of electric loads," Proceedings of the IEEE, vol. 3, no. 1, pp. 434-442, 2012.

[4] C. Guille and G. Gross, "A conceptual framework for the vehicle-to-grid (V2G) implementation," Energy Policy, vol. 37, no. 11, pp. 4379-4390, 2009.

[5] H. Hao, B. Sanandaji, K. Poolla, and T. Vincent, "A generalized battery model of a collection of thermostatically controlled loads for providing ancillary service," in Proc. of Allerton Conference on Communication, Control, and Computing, Oct 2013, pp. 551-558.

[6] B. Kirby, "Frequency regulation basics and trends," Oak Ridge National Laboratory, Tech. Rep., 2004.

[7] _ - "Ancillary services: Technical and commercial insights," Wartsila North America Inc., Tech. Rep., 2007.

[8] P. Kokotovic and A. Haddad, "Controllability and timeoptimal control of systems with slow and fast modes," IEEE Transactions on Automatic Control, vol. 20, no. 1, pp. 111-113, Feb 1975.

[9] A. Venkat, I. Hiskens, J. Rawlings, and S. Wright, "Distributed MPC strategies with application to power system automatic generation control," IEEE Transactions on Control Systems Technology, vol. 16, no. 6, pp. 1192-1206, Nov 2008.
[10] J. Mathieu, M. Kamgarpour, J. Lygeros, and D. Callaway, "Energy arbitrage with thermostatically controlled loads," in Proc. of European Control Conference, July 2013, pp. 2519-2526.

[11] M. Caramanis, I. Paschalidis, C. Cassandras, E. Bilgin, and E. Ntakou, "Provision of regulation service reserves by flexible distributed loads," in Proc. of IEEE Decision and Control Conference, Dec 2012, pp. 3694-3700.

[12] H. Hao, A. Kowli, Y. Lin, P. Barooah, and S. Meyn, "Ancillary service for the grid via control of commercial building hvac systems," in Proc. of IEEE American Control Conference, June 2013, pp. 467-472.

[13] S. Meyn, P. Barooah, A. Bušić, and J. Ehren, "Ancillary service to the grid from deferrable loads: the case for intelligent pool pumps in Florida (Invited)," in Proc. of IEEE Conference on Decision and Control, 2013.

[14] S. Koch and G. Andersson, "Assessment of revenue potentials of ancillary service provision by flexible unit portfolios," in Proc. of IEEE Power and Energy Society General Meeting, July 2012, pp. 1-8.

[15] E. Vrettos and G. Andersson, "Scheduling and provision of secondary frequency reserves by aggregations of commercial buildings," IEEE Transactions on Sustainable Energy, vol. PP, no. 99, pp. 1-15, 2015.

[16] E. Vrettos, F. Oldewurtel, and G. Andersson, "Robust energy-constrained frequency reserves from aggregations of commercial buildings," IEEE Transactions on Power Systems, vol. PP, no. 99, pp. 1-14, 2016.

[17] J. T. Hughes, A. D. Domínguez-García, and K. Poolla, "Identification of virtual battery models for flexible loads," IEEE Transactions on Power Systems, vol. PP, no. 99, pp. $1-10,2016$.

[18] A. Wood and B. Wollenberg, Power Generation, Operation, and Control. New York: Wiley, 1996.

[19] D. Bertsimas and J. Tsitsiklis, Introduction to Linear Optimization, 1st ed. Athena Scientific, 1997.

[20] S. Boyd and L. Vandenberghe, Convex Optimization. New York, NY, USA: Cambridge University Press, 2004.

[21] J. Löfberg, "Minimax approaches to robust model predictive control," Ph.D. dissertation, Linköping University, Linköping, Sweden, 2003.

[22] D. Apostolopoulou, P. Sauer, and A. Domínguez-García, "Automatic generation control and its implementation in real time," in Proc. of Hawaii International Conference on System Sciences, Jan 2014, pp. 2444-2452.

[23] "Fast response regulation signal," http://www.pjm. com/markets-and-operations/ancillary-services/ mkt-based-regulation/fast-response-regulation-signal. aspx.

[24] "PJM regulation zone preliminary billing data," http://www.pjm.com/markets-and-operations/ market-settlements/preliminary-billing-reports/ pjm-reg-data.aspx. 